Editorial

\title{
Predictor of neonatal morbidity in birth asphyxia
}

\author{
Rabindran ${ }^{1}$, Gedam DS ${ }^{2}$ \\ ${ }^{1}$ Dr. Rabindran, Consultant Neonatologist, Sunrise Superspeciality Children's Hospital, Hyderabad., ${ }^{2}$ Dr D Sharad Gedam, \\ Editor, IJMRR and associate with L N Medical college, Bhopal, MP,India.
}

Address for correspondence: Dr Rabindran, E mail: rabindranindia@yahoo.co.in

\begin{abstract}
Hypoxic ischaemic brain damage is a major cause of neonatal mortality and morbidity. Similar degrees of insult may completely spare one child and devastate another, so very sensitive prognostic parameters are needed. Conventional predictors like fetal heart rate patterns, Apgar scores, need for resuscitation and umbilical arterial blood gas analysis show variable predictive values of outcome in neonates with asphyxia.
\end{abstract}

Key words: birth asphyxia, Neonatal morbidity, Predictor of outcome

Conventional perinatal variables such as Apgar score and cord $\mathrm{pH}$ are not specific predictors of adverse outcome. The stage of encephalopathy in the first week of life is probably the single best clinical predictor of long-term outcome after acute perinatal asphyxia. However early prediction of HIE is needed for selection of newborn infants who could benefit from neuroprotective treatment like hypothermia.

Recent papers have drawn our attention to the prognostic value of lactic acidaemia in newborns with severe hypoxaemia [1]. Serum lactate levels in the first 30 minutes of life do not predict the severity of the ensuing encephalopathy. In contrast, sustained lactic acidosis is associated with severe encephalopathy on EEG and correlates with seizure burden. Plasma lactate $>9 \mathrm{mmol} / \mathrm{l}$ was associated with moderate or severe encephalopathy with a sensitivity of $84 \%$ and a specificity of $67 \%$ [2]. Cerebral lactate \& urinary lactate are correlated with neonatal outcome. LDH is a good predictor of HIE during the first $12 \mathrm{~h}$ after birth. A cut off level of $1049 \mathrm{U} / \mathrm{L}$ for LDH was the best predictor of HIE (sensitivity $100 \%$ and specificity 97\%) [3]. Salivary LDH also provided an early and accurate diagnosis of HIE and could be used as a triage tool. Degree of metabolic acidosis correlated with the neonatal neurological outcome. However it may lack sensitivity when asphyxia occurs during the early stages of labour. Hyperglycemia in the first 12 hours of life is associated with poor gross motor outcome or death in asphyxiated term infants. NRBCs can be considered as a marker of perinatal asphyxia and significantly correlate with the degree of asphyxia.

Biomarkers of neonatal hypoxic ischemic encephalopathy predictive of abnormal outcomes include glial fibrillary acidic protein, brain-derived neurotrophic factor, S100b, serum and CSF concentrations of IL-1b, IL-6, and serum neuron-specific enolase (NSE). GFAP $=/>$ than 0.15 $\mathrm{ng} / \mathrm{mL}$ was predictive of an abnormal brain MRI [4]. Higher cord plasma BDNF levels among babies with HIE had poor outcome. Urinary S100b concentrations above 1 $\mathrm{mcg} / \mathrm{L}$ predicted neonatal death with a sensitivity and specificity of $100 \%$, and concentrations were not affected by renal failure [5]. Cord blood S100b > $2.02 \mathrm{mg} / \mathrm{L}$ has a

International Journal of Medical Research and Review sensitivity of $87 \%$ and a specificity of $88 \%$ for predicting the development of moderate or severe HIE [6]. IL-1â seems to be a better predictor of HIE than TNF-alpha. Interleukin-6 concentrations in CSF after hypoxia were significantly higher in the patients with adverse outcome. Serum NSE > $40 \mathrm{mcg} / \mathrm{L}$ obtained between 4 \& 48 hours after birth can distinguish infants who have mild HIE from infants who have moderate or severe HIE [7]. Glutamate level has been shown to correlate with grade of HIE and outcome. VEGF system is up-regulated in response to placental hypoxia and is assumed to be a potential early indicator of severe birth asphyxia.

Cardiac biomarkers aid in long term neurodevelopmental outcome prediction following neonatal hypoxicischaemia. ECG changes were significantly associated with poor prognosis $(\mathrm{p}=0.001)$, with negative predictive value of $90 \%$, but poor positive predictive value of $53 \%$ \&were associated with multi-organ involvement, low Apgar score at $5 \mathrm{~min}$ and severe grade of encephalopathy. Newborns with severe neonatal hypoxic ischaemia have significantly higher serum troponin-T concentrations than other asphyxiated groups (mild to moderate) and healthy neonates on day 1 of life. The optimal cut-off value of cord troponin-I for prediction of perinatal hypoxia was $0.35 \mathrm{lg} \Lambda$ and for prediction of serious risk of early mortality was $4.6 \lg \Lambda$ [8]. Cord troponin-I is the marker with highest specificity $(86 \%)$, sensitivity (88\%), NPV $(85 \%)$, PPV $(88 \%)$ for prediction of perinatal hypoxia and was identified as the most sensitive factor for predicting early death [8]. The levels of antioxidant enzymes like lipid peroxidase can reliably and significantly predict mortality and morbidity. Determining the serum levels of oxidative stress markers and protein carbonyl can be used as predictors of immediate outcome in perinatal asphyxia.

High CPK activity was noted as a sensitive indicator of conspicuous brain damage. Serum CK-BB activity determined in cord blood between 6 and $12 \mathrm{~h}$ post-partum is an effective predictor of neurological prognosis. CSF glycine, Glutamate \& aspartate concentration correlated significantly with the severity of HIE. Hypoxanthine level is a better predictor of hypoxia-related sequelae than Apgar score or lactate level. Urinary microglobulin was Available online at: www.ijmrr.in 364 | P a g e 


\section{Editorial}

the most sensitive indicator of systemic organ injury in perinatal asphyxia. N-acetylaspartate, a marker of neuronal integrity declines after asphyxial injury, and this decline is predictive of later neurologic deficits. Cerebral ultrasound scanning was the most commonly used predictor of the outcome previously. However it is a poor predictor of outcome, with a likelihood ratio of close to 1 because of a high false positive rate. With Doppler measurements, the false positive rate was 0 but the detection rate (sensitivity) was only $23.5 \%$. MRI demonstration of involvement of subcortical gray matter structures, such as the basal ganglia and thalamus, a T1weighted signal in the posterior limb of the internal capsule are adverse prognostic markers. Abnormal intracerebral lactate levels on magnetic resonance spectroscopy within 18 hours of birth in infants with HIE and correlate with neurodevelopmental outcome. Higher $\mathrm{Glx}-\alpha / \mathrm{Cr}$ value by proton magnetic resonance spectroscopy in basal ganglia and thalamus in neonates with HIE may predict a poor outcome.

Amplitude-integrated electroencephalography (EEG) pattern and voltage was found to have a good correlation with outcome in term infants who suffered from hypoxicischemic encephalopathy. Early EEG changes (within 48 hours of age) in the acute phase is highly predictive of very unfavorable short-term outcomes. During the first 6 hours after birth, a bi-parietal aEEG is the most sensitive and specific single indicator of long-term outcome after HIE. The cerebral function monitor (CFM) has been used in term neonates to evaluate prognosis after perinatal asphyxia. Cerebral haemodynamics showing higher CBF and $\mathrm{CBV}$ on the first day of life had adverse outcomes, and a CBV outside the normal range had a sensitivity of $86 \%$ for predicting death or disability. The value of CBF velocity changes to predict poor outcome in asphyxiated infants is low 2-6 hour after asphyxia, but increases by the age of 12 hour. Because of its noninvasive, easy-touse nature, neonatal polysomnography has become an important assessment method for neurologically damaged infants. Polygraphic evidence of status epilepticus, hypovoltage, and burst suppression pattern plays an important role in predicting neurologic disabilities. The need for early prediction of outcome of birth asphyxia is particularly important because of the narrow window of effectiveness and possible side effects of neuro-protective interventions. With advanced technology, biochemical and clinical assessment it is possible to prognosticate asphyxiated babies early which helps in early intervention.

Pal et al in in his study revealed that linear relationship between levels of cardiac troponin-I and birth asphyxia.
Therefore cardiac troponin-I level may be useful in predicting the mortality and outcome in perinatal asphyxia [9].

\section{References:}

1. Cheung PY, Robertson CMT, Finer NN. Plasma lactate as apredictor of early childhood neurodevelopmental outcome ofneonates with severe hypoxemia requiring extracorporeal mem-brane oxygenation. Arch Dis Child 1996; 74: F47-503.

2. Da Silva S, Hennebert N, Denis R, Wayenberg J-L. Clinical value of a single postnatal lactatemeasurement after intrapartum asphyxia. Acta Pædiatr 2000; 89(3): 320-3. 10.1111/j.1651-2227.2000.tb01334.x

3. Mathias Karlsson, Eva Wiberg-Itzel, Ela Chakkarapani, Mats Blennow, Birger Winbladh, Marianne Thoresen. Lactate dehydrogenase predicts hypoxic ischaemic encephalopathy in newborn infants: a preliminary study. Acta Paediatrica 2010;99(8) : 1139-1144. Doi: 10.1111/j.1651-2227.2010.01802.x

4. Martha Douglas-Escobar, Michael D. Weiss. Neonatal Biomarkers of Brain Injury; NeoReviews Vol.14 No.10 October 2013 e501. doi: 10.1542/neo.14-10-e501

5. Gazzolo D, Frigiola A, Bashir M, et al. Diagnostic accuracy of S100b urinary testing at birth in full-term asphyxiated newborns to predict neonatal death. PloS one. 2009;Feb 4(2):e4298 doi: 10.1371/journal.pone.0004298

6. Qian J, Zhou D, Wang YW. Umbilical artery blood S100b protein: a tool for the early identification of neonatal hypoxicischemic encephalopathy. Eur J Pediatr. 2009;168(1):71-77. . doi: 10.3389/fneur.2012.00144 PMCID: PMC3486976

7. E Thornberg, $\mathrm{K}$ Thiringer, $\mathrm{H}$ Hagberg, I Kjellmer.Neuron specific enolase in asphyxiated newborns: association with encephalopathy and cerebral function monitor trace. Arch Dis Child Fetal Neonatal Ed. 1995 Jan; 72(1): F39-F42.

8. Tu“rker G, Babaog lu K, Go“kalp AS, Sarper N, Zengin E, Arisoy AE. Cord blood cardiac Troponin I as an early predictor of short-term outcome in perinatal hypoxia. Biol Neonate 2004; 86: 131-7.

9. Pal P, Goel M. ECG changes in birth asphyxia and its correlation with Cardiac troponin-I. Int J Med Res Rev 2015;3(4):400-403. doi: 10.17511/ijmrr.2015.i4.075.

\section{How to cite this article?}

Rabindran, Gedam DS. Predictor of neonatal morbidity in birth asphyxia. Int J Med Res Rev 2015;3(4):364-365. doi: 10.17511/ijmrr.2015.i4.085. 